\title{
CENTROS URBANOS DE AMÉRICA LATINA 1997, 2006: DISPARIDADES SALARIALES SEGÚN GÉNERO Y CRECIMIENTO ECONÓMICO
}

\section{URBAN CENTERS IN LATIN AMERICA 1997, 2006: INCOME INEQUALITIES RELATED TO GENDER DIFFERENCES AND ECONOMIC GROWTH}

\author{
Giovanni E. Reyes*
}

RESUMEN

\begin{abstract}
Uno de los rasgos de inequidad directa de género que mantienen muchos países, es el conjunto de disparidades salariales entre hombres y mujeres. El argumento central de este estudio es que las desigualdades salariales entre países tenderán a mejorar en aquellos que tienen economías más pequeñas, respecto a los de economías de grandes mercados relativos. Se hace aquí una comparación entre la inequidad salarial en países latinoamericanos en 1997 y en 2006 . A este rasgo de inequidad directa se debe agregar la inequidad indirecta, pero no menos significativa, que se tiene con las mujeres, con medidas muy influenciadas por las matrices culturales prevalecientes, tales como la tendencia aún presente, de que las mujeres estudian menos ciertas carreras vinculadas con la innovación y las tecnologías. La inequidad salarial tiende a mejorar en Latinoamérica, pero aún es necesario persistir en las mejoras. Países como Perú, Brasil y Bolivia aparecen como los de mayor inequidad salarial respecto a género.
\end{abstract}

PALABRAS CLAVE: AMÉRICA LATINA * INEQUIDADES DE GÉNERO * ECONOMÍA URBANA * INGRESO * EMPLEO

\section{ABSTRACT}

A particular case of direct inequity conditions related to gender differences is the prevailing level of unequal income in urban centers in Latin America. The existence of this phenomenon within specific regional clusters is the core argument of this study. To support this claim, this paper establishes comparative considerations from gender income inequities in Latin American urban centers, in 1997 and 2006. To this feature of direct inequity based on gender differences, it is important to add

Profesor asociado de la Universidad Nuestra

Señora del Rosario, Colombia.

giovr@yahoo.com 
other discriminate practices still alive, such as the fact that in some areas girls do not enter to study or prepare themselves, in fields more related with technology and productive innovation. Based on results from this study, Latin America as a region has shown improvements in income inequalities, but there is indisputable need for improvements. Countries such as Peru, Brazil and Bolivia appear with high standards of income differences based on gender discrimination.

KEYWORDS: LATIN AMERICAN * GENDER INEQUALITIES * URBAN ECONOMY * INCOME * EMPLOYMENT

\section{INTRODUCCIÓN}

Son varios los países que en particular, desde la década de los setenta, han establecido entre sus políticas públicas, disposiciones relacionadas con género. Entre ellos se destacan Venezuela, Brasil, México, Argentina, Uruguay y Costa Rica. Esas políticas han estado generalmente relacionadas con la dotación de alimento, nutrición y cuidado de niños, el freno a las condiciones de discriminación y acoso laboral, la mejora en la remuneración económica y participación política. En varios casos se han constituido instituciones, direcciones generales de ministerios o vice-ministerios, conformados en función de agencias que abordan la problemática de género ${ }^{1}$.

Por otra parte, en especial desde los años sesenta y setenta del siglo Xx, se ha hecho evidente una consolidación de estudios de economía y sociología para el desarrollo, enfatizando los conceptos de diversidad, desigualdad e inequidad. Esto se ha realizado no solo en función de patrones de trato y distribución de beneficios que afectan a diferentes grupos étnicos, sino muy especialmente en cuanto a relaciones de género ${ }^{2}$.

$1 \quad$ Para una mayor discusión respecto a los vínculos entre política pública, institucionalidad y condiciones de género, véase: UNDP, 2001. En particular el capítulo II, que además de la base conceptual, aborda las consideraciones sobre agencia, desarrollo y participación de género; capítulo IV: feminización de la pobreza y capítulo VII: aspectos políticos, disposiciones nacionales y participación de mujeres; también Barrera y Aguirre, 2003; Massolo, 1994 y 2005.

2 Especialmente en este aspecto, el presente documento contiene - en particular en la revisión conceptual- un resumen del trabajo que el autor
En general, existen dos tipos de discriminación contra la mujer: uno de ellos, de carácter directo, que se evidencia más en las disparidades salariales con hombres - asumiendo, como se hace en este estudio- que tal disparidad opera cuando individuos pertenecientes a ambos sexos tienen igual nivel de preparación y desempeñan la misma actividad con similar grado de productividad. Otro indicador directo, sería la preferencia en el otorgamiento de empleo a hombres sobre mujeres.

De manera indirecta, no por ello menos significativa, el factor preparación incide en las mujeres en la determinación de sus proyectos de vida. Aún es posible establecer cómo las niñas tienden a tener un tipo de educación o capacitación que hace menor énfasis en carreras asociadas con tecnología pesada y sectores ligados a innovación productiva.

El argumento central de este estudio, en términos más específicos, consiste en sostener que: si bien es cierto, los países de economía de mercado interno relativamente pequeño, pueden no crecer tan rápidamente - tal es el caso de Nicaragua u Honduras- allí se pueden evidenciar mejoras notables en los grados de menor desigualdad entre salarios de mujeres respecto a hombres.

El principal objetivo de este artículo se centra en dos aspectos:

Primero, hacer una revisión conceptual sobre diferentes metodologías en donde se ha considerado la problemática de

realizará para la Organización de Naciones Unidas, en preparación para un Seminario Internacional sobre Empleo y Política Pública; se trata de documentos de apoyo para la preparación del Informe de Desarrollo Humano Mundial. 
segregación y discriminación. Se entenderá aquí que la segregación en términos de división, no implica componentes valorativos culturales. En cambio, la discriminación esencialmente establece componentes psico-sociales, que son parte del imaginario colectivo, por lo general con tendencias peyorativas. En muchos casos, es necesario dar un tratamiento desigual a grupos o individuos, a fin de introducir el concepto de equidad, entendida esta como el trato justo de las diferencias entre individuos o agrupaciones ${ }^{3}$.

Segundo, realizar un estudio comparativo sobre cifras de diferentes países latinoamericanos y caribeños en relación con las inequidades existentes en términos de remuneraciones económicas. Específicamente, lo que se trata de realizar es un contraste con las disparidades salariales entre hombres y mujeres, en 18 países de Latinoamérica y el Caribe, con datos de 1997 y 2006.

A partir de lo anterior, se considera el crecimiento económico de los diferentes países. Se estudia el crecimiento económico anual que han tenido en los últimos 10 años, de 2000 a 2009. Con base en los datos de desigualdad en salarios en su connotación dinámica, es decir, las variaciones que presentaron los valores correspondientes y el crecimiento económico acumulado de los últimos diez años, se establece un estudio de elasticidades —variación

3 Una mayor discusión sobre teoría de la segregación/discriminación, en Olson, Steve. Mapping human history: discovering the past through our genes. New York: Houghton Mifflin, 2002; Loury, Glenn. The anatomy of racial inequality. Cambridge, MA: Harvard University Press, 2002 y también de este autor: su trabajo presentado en la Conferencia Mundial contra el Racismo: Racial stigma: toward a new paradigm for discrimination theory. Sudáfrica, 2001. Se trata de un trabajo publicado por la Universidad de Boston. Diferenciaciones y dinámicas propias de la segregación y la discriminación son enunciados en este aporte, el mismo emerge con estudios de caso de Estados Unidos, pero su planteamiento conceptual y metodología pueden ser generalizables a otros países. de desigualdad salarial por uno por ciento de variación en crecimiento.

El desarrollo de estudios de este tipo requiere de actualización permanente. Pueden llegar a ser medios que contribuyan al monitoreo o evaluación sistemática de procesos de gestión, o elementos de criterio para la formulación y el establecimiento de políticas públicas ${ }^{4}$.

\section{ASPECTOS CONCEPTUALES}

Se consideraron como más pertinentes, para las finalidades de ilustración metodológica de este artículo, los aportes de cuatro trabajos sobre segregación y discriminación: Arcand y D'Hombres (2004); Demurger, Fournier y Yi (2005); Esteve-Volart (2004) y Graham (2002). Esta última obra está enfocada en la identificación de metodologías e indicadores para detectar las dinámicas de segregación o discriminación ${ }^{5}$.

En el estudio de Arcand y D'Hombres (2004) se identifican tres tipos de discriminación directa que pueden operar en una sociedad en general, discriminación por: (i) acceso a

4 Como instrumento de evaluación sistemática o monitoreo, esta técnica está siendo utilizada por algunas organizaciones de gobierno, en cuanto a medir el alcance y probabilidad de alcanzar las metas del milenio. Un estudio de caso, a manera de ilustración, se realizó en Filipinas y se aplicó a la evaluación de políticas dirigidas especialmente a: (i) reducción de pobreza, (ii) reducción de indicadores de hambruna, especialmente en niños menores de cinco años, (iii) aumento de accesibilidad a agua potable, (iv) cobertura de educación primaria y $(v)$ eliminación de disparidades de género. Al respecto, véase: Collas-Monsod, Solita et ál."Philippines' progress towards the millenium development goals: geograpphical and political correlates of subnational outcomes". Journal of Human Development 5 (1). New York: UNDP, March 2004: 121-128. Especialmente, la introducción y primera parte.

5 Véase: Arcand, D’Hombres. Sección II: Metodología y la sección III: Análisis Empírico. 2004: 3-12; Demurger, Fournier y Yi. Sección II: Metodología. 2005: 3-6; Esteve-Volart. Sección I: Revisión de literatura y la sección IV: Modelos. 2004: 2-5 y 9-31, respectivamente; Graham. 2002. Especialmente págs. 59-65, 71-82 y 84 . 
empleo, (ii) salarios y (iii) ocupacional por grandes líneas o áreas de producción. Existiría otro tipo de discriminación, más indirecta, pero no menos importante, la que se refiere a los estudios diferenciados entre niños y niñas.

El modelo general que utilizan, se basa en distinguir en las condiciones de Brasil, durante los noventa, entre los factores de productividad por una parte y por la otra, los efectos de la discriminación. Para su estudio, separan a la población blanca de los afrobrasileños. Para efectos de determinar la brecha en las tasas de empleo, el mercado de trabajo se estudia con base en los siguientes modelos:

$$
\begin{aligned}
\stackrel{-w}{P}-\stackrel{-b}{P}= & {\left[{ }^{-} \varphi\left(\delta^{*} X^{w}\right)-\bar{\varphi}\left(\delta^{*} X^{b}\right)\right] } \\
& +\left[\bar{\varphi}\left(\delta^{w} X^{w}\right)-\bar{\varphi}\left(\delta^{*} X^{w}\right)\right] . . \\
& +\left[{ }^{-} \varphi\left(\delta^{*} X^{b}\right)-\bar{\varphi}\left(\delta^{*} X^{b}\right)\right]
\end{aligned}
$$

Donde:

$\stackrel{-i}{\mathrm{P}}=$ es la probabilidad que tienen de empleo los integrantes del grupo $\mathrm{i}$

$\bar{\varphi}()=$. es el promedio predecible de probabilidad de empleo

$\mathrm{X}^{\mathrm{i}}=$ es el vector que determina las dotaciones de cada grupo

$w=$ identifica al grupo blanco

$\mathrm{b}=$ identifica al grupo afrobrasileño

$\delta^{*}=$ vector de coeficientes de empleo con no discriminación

$\delta^{i}=$ vector de coeficientes detectados (con discriminación)

Este modelo se ha adaptado de una metodología previa que se utilizó para estudiar patrones de segregación/discriminación, denominada metodología Oaxaca (1973,
1994). Es interesante determinar que la descomposición de factores en función de establecer brechas de productividad y brechas de discriminación, se fundamenta en la siguiente descomposición:

$$
\left[\bar{\varphi}\left(\delta^{*} X^{w}\right)-\bar{\varphi}\left(\delta^{*} X^{b}\right)\right]
$$

La cual sería el diferencial debido a productividad $y$

$$
\begin{aligned}
& {\left[{ }^{-} \varphi\left(\delta^{w} X^{w}\right)-\bar{\varphi}\left(\delta^{*} X^{w}\right)\right]+} \\
& {\left[\bar{\varphi}\left(\delta^{*} X^{b}\right)-\bar{\varphi}\left(\delta^{*} X^{b}\right)\right]}
\end{aligned}
$$

Que sería la brecha por causa de la discriminación entre grupos blanco y afrobrasileño.

Los resultados de este estudio mostraron que los blancos tienen ventajas significativas en el acceso al empleo con respecto a los afrobrasileños. Sin embargo, la discriminación mayor se evidenció respecto a los salarios. También fue importante una conclusión en función de la cual se pudo argumentar con base empírica, que muchas de las desventajas de capacidades o dotaciones de los grupos, se derivaban significativamente, del acceso que se tenía respecto a la educación.

En el trabajo de Demurger, Fournier y Yi (2005), se estudia la discriminación por motivo de género en el aspecto laboral, que ha ocurrido a raíz de las políticas de liberación comercial y económica en China. Especialmente a partir de los cambios de $1978^{6}$.

$6 \quad$ Sobre la situación estricta de diferenciales de salario por motivo de género en China, además, son interesantes, especialmente por motivos de formulación de indicadores, los estudios de Dong et ál., 2004: 979-998; Ho et ál., 2002: 659-688; Meng, 1995: 136-155 y de Rozelle, 2002:157-179. 
La muestra del estudio se refirió a la población económicamente activa urbana en China durante la década de los ochenta y noventa del siglo Xx. Nótese que en el estudio anterior, la discriminación fue basada en los diferenciales entre grupos étnicos; aquí en diferencias salariales entre géneros.

Los modelos fundamentales utilizados se basaron primero, en la caracterización de los salarios a nivel individual, sin comparaciones:

$$
w_{i, g}^{t}=W\left(x_{i, g}^{t} ; \beta_{g}^{t}\right)
$$

Donde:

$\mathrm{W}_{\mathrm{i}, \mathrm{g}}^{\mathrm{t}}=$ ingresos de un individuo i con género g, en un tiempo $t$

$\chi_{\mathrm{i}, \mathrm{g}}^{\mathrm{t}}=$ características individuales en tiempo $\mathrm{t}$ $\beta_{\mathrm{g}}^{\mathrm{t}}=$ coeficiente de observaciones empíricas de ingresos con base en características del género g.

A partir de esta caracterización individual, se identifica la brecha de ingresos incluyendo comparaciones en tiempo:

$$
\Delta_{t}^{T} \mathrm{D}_{1}=\left\lfloor W_{h}^{T}-W_{m}^{T}\right\rfloor\left\lfloor W_{h}^{t}-W_{m}^{t}\right\rfloor
$$

En (3) se muestra la comparación entre brechas entre géneros en los tiempos $\mathrm{T}$ y $\mathrm{t}$; T equivaldría a $(\mathrm{t}+\mathrm{n})$. W son los salarios $y \mathrm{~h} / \mathrm{m}$ son los distintivos para hombre $y$ mujer, respectivamente. A lo anterior se agrega, como un tercer nivel de comparaciones, las brechas, pero en términos de observadas $y$ las simuladas:

$$
\begin{gathered}
\Delta_{i}^{T} \mathrm{D}_{2}=\left\lfloor W\left(x_{i, h}^{T} ; \beta_{h}^{T}\right)-W\left(x_{i, m}^{T} ; \beta_{m}^{T}\right)\right\rfloor \\
\left\lfloor W\left(x_{i, h}^{T} ; \beta_{h}^{t}\right)-W\left(x_{i, m}^{T} ; \beta_{m}^{t}\right)\right\rfloor \ldots .
\end{gathered}
$$

En (4) el primer corchete se referiría a la brecha determinada en (3), a la que se le agrega la determinación de la brecha detectada en función de la simulación de brechas de poblaciones observadas en tiempos $\mathrm{T}$ y $\mathrm{t}$, que corresponderían al segundo corchete.

Este estudio sobre diferencias salariales en centros urbanos de China, basado en desigualdades de género, encontró con significativo grado de diferencia estadística que las reformas habían repercutido negativamente en los ingresos de las mujeres. No obstante, se determinó que esas brechas tendían a tener menores márgenes de diferencia, al pasar de ser 18,7\% en 1988 a 17,7\% en 1995.

La discriminación parecía más notable, durante el tiempo estudiado, en las empresas de inversión china que en aquellas donde dominaba el capital extranjero. Por último, la discriminación era más evidente, como es el caso de otras sociedades, en los trabajos que tenían bajas remuneraciones; es decir, que las brechas salariales debido a diferencias de género tendían a ser menores a medida que los salarios y las posiciones se elevaban dentro de la jerarquía de las empresas.

En el trabajo de Esteve-Volart (2004), el tema de estudio es la discriminación también de género en términos de empleo y salarios, en el contexto de crecimiento económico de India, durante el período 1961-1991. Los modelos que utiliza se basan en comparar las condiciones de oferta $y$ demanda de mano de obra con diferentes niveles o grados de capacitación.

Se estudian allí varias divisiones específicas que ocurren en el mundo laboral: (i) división entre trabajo de administradores $y$ de operarios, (ii) equilibrio de mercados laborales totales sin y con discriminación, (iii) equilibrios de mercados laborales para administradores con y sin discriminación y (iv) equilibrios de mercados laborales para operarios con $y$ sin discriminación.

Esteve-Volart concluye que las diferentes formas de discriminación, no solo son manifestaciones de inequidad en el mundo laboral, sino que las mismas constituyen frenos 
a la eficiencia productiva; contribuyen a mantener a la sociedad en condiciones de ineficacia, dentro del área por debajo de la frontera de producción posible. La discriminación hace disminuir los ingresos per cápita y distorsiona la distribución laboral de talentos. Se discuten también los contenidos de reformas sociales $y$ hasta qué punto las mismas pueden ser sostenibles y redundar en el mantenimiento de patrones de discriminación contra mujeres.

El estudio de Graham (2002) contiene un mayor énfasis en la movilidad de ingresos y en las brechas que se pueden mantener durante el tiempo. Se analizan las relaciones entre movilidad social basada en ingresos y aspectos de equidad. Los indicadores tratan de diferenciar dos medidas de inequidad: (i) la movilidad de ingresos durante el período de vida profesional, respecto a diferentes carreras, consideradas las mismas de manera individual y (ii) la inequidad de ingresos intergeneracionales ${ }^{7}$.

Lo que se desea destacar aquí, es la utilización especialmente de tablas o cuadros de resultados, los cuales están relacionados con el uso de matrices de Markov para determinar escenarios de movilidad y distribución.

Siguiendo un modelo simple de utilización de cadenas de Markov, se tendrían los siguientes elementos: una matriz base, una matriz de transición o factores y una matriz de resultados. Esta última es la que utiliza Graham para caracterizar la movilidad de ingresos de la población.

Ilustrando rápidamente, la metodología del uso de cadenas de Markov se tiene:

$7 \quad$ Específicamente para teoría y aplicaciones de indicadores de inequidad, véase Schwartzman, Lisa. "Liberal rights theory and social inequality: a feminist critique". Hypatia 14 (2). Spring 1999: 26-47; Harding, Sandra. Science and social inequality. University of Illinois Press, 2006; Hurst, Charles. "An introduction to the study of social inequality". Capítulo I. y "Sex and gender inequality". Capítulo IV. Social inequality: forms, causes and consequences. New York: Allyn \& Bacon, 2006: 3-12 y 60-86, respectivamente.

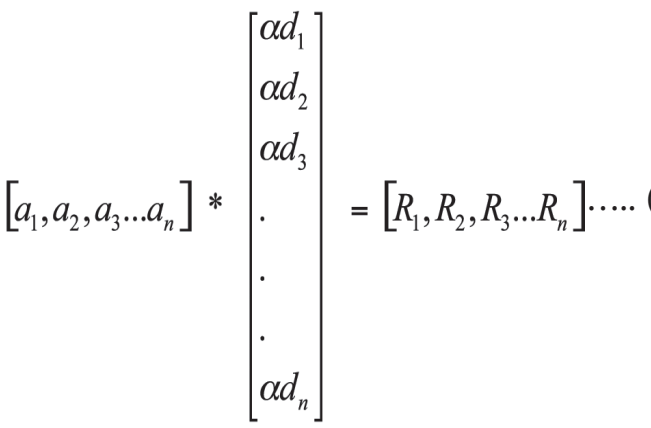

En (5), se representan las matrices mediante formas vectoriales, indicando la primera (de coeficientes a) las condiciones que se tienen en el escenario base, en el tiempo t. La matriz de coeficientes $\alpha d_{\mathrm{i}}$ representa la matriz de transición.

Por ejemplo, la matriz de coeficientes $\alpha d_{\mathrm{i}}$ podría proporcionar la información sobre los porcentajes de cambio de empleo, que se observan o esperan entre trabajadores con respecto a los diferentes sectores de producción. Finalmente, la matriz de coeficientes $\mathrm{R}_{\mathrm{i}}$, daría el resultado; es decir, el producto de multiplicación de las dos matrices anteriores. Esta última matriz serviría para determinar el escenario resultante influenciado por la movilidad o transformación evidenciada ${ }^{8}$.

En particular, en lo que se refiere a movilidad, Graham utiliza matrices de resultados en las cuales muestra la situación en un determinado año base, por quintiles de población respecto a ingreso y luego, establece cómo integrantes de cada quintil se han movilizado o no en un año posterior. En el Cuadro 1, se muestra un ejemplo específico de

8 Ampliaciones, discusión conceptual y modelos aplicativos en: Govan, A. y Meyer, C. Applying theory of Markov chains to the problem of ranking. North Carolina State University, 2006; Stewart, G. "On the perturbation of Markov chains". Numerische Mathematik 65. 1993: 135-141; Kesidis, G. y Walrand, J. "Relative entropy between Markov transition rate matrices". Information Theory 39 (3). 1993: 1056-1057; Neuts, Marcel. Multivariate semi-Markov matrices. Purdue University: Departamento de Estadística, 1999. En: $<$ http://stinet.dtic.mil/oai/oai?\&verb=getRecord\& metadataPrefix $>$ 
CUADRO 1

PERÚ: MOVILIDAD DE GRUPOS SOCIALES

QUINTILES SEGÚN NIVELES DE INGRESO

1991, 1997

\begin{tabular}{ccccccc}
\hline $\begin{array}{c}\text { QUINTILES } \\
1991\end{array}$ & 1 & 2 & 3 & 4 & 5 & TOTAL \\
\hline 1 & 41 & 30 & 19 & 11 & 00 & 100 \\
2 & 26 & 33 & 15 & 19 & 07 & 100 \\
3 & 22 & 15 & 30 & 22 & 11 & 100 \\
4 & 11 & 19 & 22 & 26 & 22 & 100 \\
5 & 00 & 04 & 15 & 22 & 59 & 100 \\
TOTAL & 100 & 100 & 100 & 100 & 100 & 100 \\
\hline
\end{tabular}

Fuente: Graham, Carol. "Mobility, opportunity and vulnerability: the dynamics of poverty and inequality in a global economy". Journal of Human Development 3 (1). New York. UNDP, 2002: 61.

Graham utilizado para Perú, en donde se comparan los años de 1991 y 1997.

La información en el Cuadro 1, se prestaría para hacer notables interpretaciones sobre la movilidad social, causales y resultados en el contexto de Perú durante los años noventa. No obstante, lo que se desea puntualizar, es por ejemplo, que el quintil 1 en 1991, retuvo un $41 \%$ de sus integrantes en tal situación, sin movilidad a otros quintiles cuando se evalúan las condiciones de 1997.

Ese mismo quintil 1 tuvo un $30 \%$ de sus integrantes que se movieron al quintil 2, 19\% al 3, 11\% al 4 y ninguno al quintil 5 de ingresos, para 1997. Cuando vemos por ejemplo, el quintil 5, la evidencia da base para indicar que un 59\% de sus integrantes logró permanecer en este nivel de ingreso y que 41\% descendió a otros quintiles; por ejemplo, un $22 \%$ se ubicó en el quintil 4, en 1997.

Este tipo de matrices de resultados pueden estudiarse en función de los rasgos de centralidad, descentralización, formación de posiciones o de "cliques" (agrupaciones que tienen una fuerte relación entre sus miembros, pero débil relación con otras unidades o sujetos). Estas caracterizaciones cuando se formulan utilizando las mencionadas cadenas de Markov $y$ las circunstancias de contexto, permiten realizar identificación de escenarios, lo que ayuda a establecer prospecciones ${ }^{9}$.

De manera complementaria, el modelo de Stolper-Samuelson, permite explicar la conformación de tendencias dinámicas, entre los sujetos y sus relaciones, proyecciones que se relacionan con patrones de segregación o discriminación. Este teorema se ha utilizado

$\overline{9} \quad$ Las interpretaciones completas de esta matriz de resultados serían motivo de un análisis de redes, lo que está fuera del alcance de este estudio. Ampliaciones sobre este tema, en: Scott, John. Social network analysis. London. SAGE Publications, 2000: 38-53, 82-99 y 100-121. Especialmente, el capítulo III: Handling relational data; capítulo V: Centrality and centralization y Capítulo VI: Components, cores and claques. Wasserman, Stanley y Galaskiewicz, Joseph (eds.). Advances in social network analysis: research in the social and behavioral sciences. London: SAGE Publications, 1994; Carrington, Peter y Scott, John (eds.). Models and methods in social network analysis: structural analysis in the social sciences. New York: Cambridge University Press y en Newman, Mark y Barabasi, Albert. The structure and dynamics of networks. New York: Cambridge University Press, 2001. 
en economía internacional, en particular, para identificar dinámicas que ocurren en intercambios de comercio internacional.

El postulado teórico de Wolfgang Stolper y Paul Samuelson fue publicado en 1941, el cual se derivó de la consideración del teorema de Heckscher-Ohlin y permite establecer adaptaciones más generalizadas o flexibles ${ }^{10}$.

Modelos más sofisticados basados en Stolper-Samuelson, pueden identificar patrones de distribución de ingresos dentro de grupos particulares de trabajadores, pertenecientes a diversas industrias ${ }^{11}$.

$10 \quad$ El teorema de Heckscher-Ohlin establece, en resumen, que la causa primaria del comercio reside en el diferencial de precios relativos de los bienes producidos en los países que intervienen, el cual se explica por diferencias en precios relativos de factores, fundamentalmente por su oferta relativa. Un país tiene ventaja comparativa en aquel bien que utiliza intensivamente el factor abundante. De acuerdo con este teorema, se esperaría un incremento de exportaciones intensivas en mano de obra no calificada y recursos naturales en países con bajos grados de desarrollo que tengan actividad exportadora más relacionada con el sector primario. Ampliaciones en Anderson, S. y Cavanagh, J. Field guide to the global economy. New York: The New Press, 2000; una referencia al caso venezolano en Baptista, A. (ed.). El pensamiento económico y su relevancia en el mundo de hoy. Caracas, Venezuela: Banco Central de Venezuela, 2002. Gilpin, Robert. Global political economy. Princeton: Princeton University Press, 2001.

11 La contribución de Stolper-Samuelson está relacionada también con el teorema de equiparación del precio de factores, el que establece que independientemente de la movilidad de factores de producción que exista, los precios de los mismos tenderán a nivelarse entre países que no tengan diferencias substantivas en tecnología. El trabajo inicial donde se publicó el teorema fue Stolper, W.F. y Samuelson, P.A. "Protection and real wages". Review of Economic Studies 9. 1941: 58-73. Ampliaciones en: Marjit, Sugata y Beladi, Hamid. "The Stolper-Samuelson theorem in a wage differential framework". Japanese Economic Review 53. 2002:177-181; Lloyd, Paul. "Generalizing the Stolper-Samuelson theorem: a tale of two matrices". Review of International Economics 8 (4). November 2000.
En Latinoamérica, sí existen evidencias en cuanto a que las mujeres tendrían los empleos más precarios. Se abordará como evidencia, la participación según género que existe en la región, en cuanto a pertenecer a los sectores formales e informales de la economía de diferentes países.

Los datos han sido dados a conocer por la Comisión Económica para América Latina (CEPAL), el Banco Interamericano de Desarrollo (BID) y la Organización de Naciones Unidas (ONU). El tema del documento es una evaluación de las evidencias que se tienen en Latinoamérica respecto al cumplimiento de los Objetivos de Desarrollo del Milenio (ODM) ${ }^{12}$.

Como se recordará, los ODM constituyeron un acuerdo que en septiembre de 2000, fijaron los Jefes de Estado del mundo, en una histórica reunión en la Organización de Naciones Unidas en Nueva York. El gran objetivo es alcanzar esos objetivos para el 2015. Una de esas metas es lograr condiciones de equidad de género en los empleos.

Los datos referidos hacen una comparación a nivel de hombres y mujeres en los mercados informales, tanto en lo urbano como en lo rural. Uno de los primeros rasgos importantes es que en todos los países, la informalidad es mayor en el medio rural que en el urbano (véase Cuadro 2).

12 Véase: Cicowiez, Martín et ál. Brechas urbanorurales y objetivos de desarrollo del milenio en América Latina y el Caribe. Buenos Aires: Centro de Estudios Distributivos, Laborales y Sociales, Universidad Nacional de La Plata, PNUD. 2006, especialmente capítulo II: "Caracterización socioeconómica" y capítulo V: "Igualdad entre géneros"; págs. 39-54 y 93-106, respectivamente. 
CUADRO 2

GÉNERO E INFORMALIDAD EN LATINOAMERICA:

ENFOQUE URBANO-RURAL

\begin{tabular}{lcccccc}
\hline \multirow{2}{*}{ PAÍ́s } & \multicolumn{2}{c}{$\%$ DE MUJERES } & \multicolumn{2}{c}{ \% DE HOMBRES } & $\begin{array}{c}\text { \% DE } \\
\text { POBLACIÓN } \\
\text { RURAL }\end{array}$ & $\begin{array}{c}\text { AÑO DE } \\
\text { ENCUESTA }\end{array}$ \\
\cline { 2 - 4 } Bolivia & RURAL & URBANO & RURAL & URBANO & RUn \\
Brasil & 92 & 67 & 79 & 50 & 38 & 2002 \\
Chile & 84 & 47 & 78 & 37 & 17 & 2002 \\
Costa Rica & 52 & 40 & 52 & 26 & 12 & 2000 \\
Rep. Dom. & 57 & 38 & 45 & 29 & 39 & 2001 \\
Ecuador & 49 & 39 & 61 & 43 & 41 & 1997 \\
El Salvador & 85 & 56 & 70 & 37 & 39 & 1998 \\
Guatemala & 85 & 49 & 62 & 36 & 40 & 2002 \\
Honduras & 89 & 56 & 60 & 35 & 53 & 2000 \\
Jamaica & 68 & 66 & 77 & 52 & 53 & 2003 \\
México & 79 & 54 & 84 & 66 & 48 & 1999 \\
Nicaragua & 77 & 60 & 73 & 47 & 23 & 2002 \\
Panamá & 65 & 33 & 65 & 44 & 43 & 2001 \\
Paraguay & 91 & 61 & 80 & 50 & 44 & 2002 \\
Perú & 91 & 62 & 75 & 44 & 25 & 2002 \\
\hline
\end{tabular}

Fuente: Agrupación propia, basada en Cicowiez, Martín et ál. Brecha urbana-rural y los objetivos de desarrollo del milenio en América Latina. 2006: 50 y 173.

En toda la región, las mujeres son las que más están ocupadas en la informalidad. Este rasgo general, no obstante, cuenta con dos excepciones. Solamente en República Dominicana y Jamaica, los hombres son más numerosos, tanto en lo rural como en lo urbano, que las mujeres ocupadas en ese espacio económico. Quizá pueda existir alguna discrepancia metodológica que pueda afectar la compatibilidad de los datos; sin embargo, esto no altera la tendencia general en la región.

Se evidencia que en tres países, casi todas las mujeres trabajadoras del medio rural lo hacen en el sector informal. Son los casos de Bolivia, Paraguay y Perú; en esas naciones un $91 \%$ de las mujeres del área rural se ocupan en las llamadas actividades de la economía subterránea o marginal. Otros países donde más del $80 \%$ de las mujeres rurales se ocupa en esos puestos de trabajo precarios, son: Brasil, Ecuador, Guatemala, Honduras y México. El valor más bajo de mujeres rurales en lo informal lo muestra República Dominicana con un 49\%.

Cuando se observa la condición de mujeres en mercados informales en el medio urbano, las cifras tienden a ser menores que los valores mostrados en el medio rural. Sin embargo, los datos no son alentadores. Los casos más altos se centran en Bolivia (67\%), Honduras (66\%) y Perú (62\%). Los países que muestran el menor porcentaje regional de mujeres trabajando en el sector informal urbano, son Panamá (33\%), Costa Rica (38\%) y República Dominicana (39\%).

En general, uno de los más grandes problemas que tiene la región latinoamericana, es la creación de empleos estables, con salarios decorosos y con prestaciones laborales. Ese es el rasgo por excelencia que vincula —además 
de la seguridad social y la asistencia directa que brindan los gobiernos- al crecimiento económico con el desarrollo. El empleo es el eslabón que posibilita que los beneficios de la economía a nivel macro puedan repercutir en los sectores mayoritarios de los países, que por lo general, dependen de un salario, para mantener $y$ aumentar su nivel de vida.

En Latinoamérica, el desempleo abierto persiste en manifestarse en casi $9 \%$. Es un nivel alto; pero el mayor desafío se encuentra precisamente con la recurrencia que se tiene en cuanto al subempleo, al empleo que no es estable, que se ubica en los obscuros circuitos de la economía informal.

En otros estudios, el sector informal se manifestaba como responsable de casi 1200 millones de millones de dólares, eso es aproximadamente un $50 \%$ del total anual de producción económica de Latinoamérica. Es allí en donde predomina la ocupación de las mujeres. Existe evidencia que se está manteniendo una condición de inequidad en los salarios para las mujeres y que tal circunstancia, manifiesta círculos viciosos de comportamiento ${ }^{13}$.

\section{METODOLOGÍA}

En primer lugar, lo que se hizo fue establecer una comparación entre las condiciones de desigualdad salarial entre hombres y mujeres en 1997 y en 2006, en 16 países de América Latina y el Caribe. En ambos casos, se determinaron análisis por agrupaciones (cluster analysis). Se estableció asimismo, una matriz comparativa

13 Ampliaciones en Hurtado, Osvaldo. Elementos para una reforma politica en América Latina. Washington DC: BID, Departamento de Desarrollo Sostenible, 2005; Banco Interamericano de Desarrollo (BID). Informe de progreso económico y social. Washington, DC: BID, 2003; Abreu, Daniel. "Empleo digno y productivo para enfrentar la pobreza y desigualdad en América Latina”. Revista Latinoamericana de Desarrollo. 2006. En: $<$ http://www.revistadesarrollohumano.org/boletin/enero_06>. Una última fuente es el Informe de Desarrollo Humano para América Latina y el Caribe, véase: Programa de Naciones Unidas para el Desarrollo (PNUD). Informe de Desarrollo para América Latina y el Caribe: Desigualdad e Inequidades. México, DF: PNUD, 2010. entre los integrantes de los grupos para ambos años, en donde se evidencia la movilidad que se hizo presente.

A continuación, se realizó un estudio respecto al crecimiento económico acumulado de 2000 a 2009 . A partir de allí, se realizó una caracterización de países también agrupados en rangos o clusters.

En una tercera fase del procesamiento de datos, se realizaron pruebas de regresión lineal entre las variables. Se determinaron las elasticidades de inequidad en función de crecimiento económico. Se mantuvo el enfoque de estudio comparado, identificando en sentido actualizado $y$ de tendencias, el comportamiento de las variables.

La inclusión de un análisis de regresión múltiple es una aproximación cuantitativa del planteamiento de investigación con respecto a impactos de condicionantes. No se trata de determinación estricta de causalidad, pero sí de contar con mayores elementos de caracterización de variables. Esto último es importante subrayarlo a raíz de los problemas de inferencia que se tendrían con el manejo de grados de libertad en la regresión múltiple que se ha desarrollado.

\section{RESULTADOS Y SU DISCUSIÓN}

Recientemente, la Comisión Económica para América Latina y el Caribe (CEPAL) ha dado a conocer no solo las condicionantes sociales generales para la región, sino en particular, las disparidades de salarios entre hombres y mujeres en centros urbanos.

La información se ha recabado basándose en estudios directos de los países y se supone que las disparidades operan cuando tanto hombres como mujeres tienen: (i) igual preparación para el desempeño de los cargos que se estudian y (ii) igual productividad. Se trataría entonces de evidencia sobre disparidad o discriminación salarial ${ }^{14}$.

$14 \quad$ Véase: Comisión Económica para América Latina y el Caribe (CEPAL). Panorama social de América Latina 2009. Santiago de Chile, Chile: CEPAL, 2009 y de ella misma, el documento, América Latina frente al espejo: dimensiones objetivas y subjetivas de la inequidad social y el bienestar en la región, 2010. 
El Cuadro 3 da a conocer los porcentajes de salarios que tienen las mujeres respecto a los hombres en centros urbanos de diferentes países latinoamericanos, tanto en 1997 como en 2006. El cuadro presenta una agrupación de naciones en las cuales se han establecido quintiles, que van desde el grupo de valores altos los de menor disparidad- a valores más bajos —de mayor disparidad ${ }^{15}$.

15 En función de aspectos metodológicos y conceptuales, respecto a la caracterización de disparidades $y$ discriminación, relacionada con aspectos directos de género e indirectos, relacionados con
En 1997, Costa Rica y Argentina mantenían altos estándares relativos en la región, en cuanto

educación y procesos de capacitación, véanse: Salomone, Rosemary. Same, different, equal. New Haven, New Jersey: Yale University Press, 2005; Krieger, Nancy. Emboying inequality. London: International Health Services, 2005; Klein, Susan. Achieving gender equity. Mahwah, New Jersey: Lawrence, Eribaum Inc., 2007; Datnou, Amanda. Gender in policy and practice. New York: Routledge Farlner, 2002; Gill, Judith. Beyond the great divide. Sydney, Australia: University of New South Wales Press, 2004 y Romesburg, Charles. Cluster analysis for researchers. London: Routledge, 2004

CUADRO 3

AMÉRICA LATINA 1997, 2006:

DISPARIDADES EN LOS SALARIOS URBANOS BASADOS EN DIFERENCIAS DE GÉNERO

\begin{tabular}{|c|c|c|c|c|c|c|c|}
\hline \multicolumn{4}{|c|}{1997} & \multicolumn{4}{|c|}{2006} \\
\hline GRUPO & PAÍS & $\begin{array}{c}\% \text { SALARIO } \\
\text { DE MUJERES }\end{array}$ & $\begin{array}{c}\text { PROMEDIO } \\
\text { DE } \\
\text { GRUPO }\end{array}$ & GRUPO & PAÍS & $\begin{array}{c}\% \text { SALARIO } \\
\text { DE MUJERES }\end{array}$ & $\begin{array}{c}\text { PROMEDIO } \\
\text { DE } \\
\text { GRUPO }\end{array}$ \\
\hline \multirow{2}{*}{ Alto } & COS & 93 & \multirow{2}{*}{92} & \multirow{4}{*}{ Alto } & VEN & 94 & \multirow{4}{*}{93} \\
\hline & ARG & 90 & & & ECU & 93 & \\
\hline \multirow{5}{*}{$\begin{array}{l}\text { Moderado } \\
\text { alto }\end{array}$} & RED & 88 & \multirow{5}{*}{86} & & $\cos$ & 91 & \\
\hline & ECU & 86 & & & $\mathrm{HON}$ & 91 & \\
\hline & VEN & 86 & & & $\mathrm{COL}$ & 89 & \multirow{4}{*}{89} \\
\hline & ELS & 84 & & Moderado & ELS & 89 & \\
\hline & PER & 84 & & alto & PAN & 89 & \\
\hline \multirow{3}{*}{ Intermedio } & PAN & 83 & \multirow{3}{*}{81} & & NIC & 88 & \\
\hline & $\mathrm{COL}$ & 82 & & \multirow{2}{*}{ Intermedio } & ARG & 85 & \multirow{2}{*}{84} \\
\hline & BOL & 79 & & & URU & 84 & \\
\hline \multirow{6}{*}{$\begin{array}{l}\text { Moderado } \\
\text { bajo }\end{array}$} & $\mathrm{HON}$ & 77 & \multirow{6}{*}{76} & \multirow{4}{*}{$\begin{array}{l}\text { Moderado } \\
\text { bajo }\end{array}$} & $\mathrm{CHL}$ & 83 & \multirow{4}{*}{82} \\
\hline & PAR & 77 & & & GUA & 83 & \\
\hline & MEX & 76 & & & RED & 82 & \\
\hline & URU & 76 & & & PAR & 81 & \\
\hline & CHL & 74 & & \multirow{4}{*}{ Bajo } & MEX & 80 & \multirow{4}{*}{79} \\
\hline & GUA & 74 & & & BOL & 79 & \\
\hline \multirow{2}{*}{ Bajo } & BRA & 70 & \multirow{2}{*}{69} & & BRA & 79 & \\
\hline & NIC & 68 & & & PER & 77 & \\
\hline
\end{tabular}

Notas: ARG: Argentina; BOL: Bolivia; BRA: Brasil; CHL: Chile; COL: Colombia; COS: Costa Rica; ECU: Ecuador; ELS: El Salvador; GUA: Guatemala; GUY: Guyana; HON: Honduras; JAM: Jamaica; MEX: México; NIC: Nicaragua; PAN: Panamá; PAR: Paraguay; PER: Perú; RED: República Dominicana; TYT: Trinidad y Tobago; URU: Uruguay; VEN: Venezuela.

Fuente: Comisión Económica para América Latina y el Caribe (CEPAL). América Latina frente al espejo: dimensiones objetivas y subjetivas de la inequidad social y el bienestar en la región. Santiago de Chile, Chile: CEPAL, 2010. 
a la proporción de ingresos de mujeres respecto a hombres. La situación tiende a cambiar para 2006, mostrando especialmente cierta caída en Argentina, pasando el indicador de $90 \%$ a $85 \%$.

En general, en 1997, el promedio de ingreso de las mujeres era de $80 \%$ respecto a los hombres y tal situación mejoró alcanzando $85 \%$ en el 2006. En la mayoría de países, las condiciones mejoran, en particular, en las naciones que tenían indicadores más bajos; pero se estanca en el caso de Bolivia, en donde prácticamente no se evidencia mejora.

En 1997, la desviación estándar de los datos daba por resultado 6,9 y para el 2006, el indicador llegó a 5,2; con ello se demostraría que no solo los datos para la región en promedio mejoraron, sino que tendieron a ser menos dispersos - esta situación se ratifica aún más al observar que el coeficiente de variación pasó de $8,6 \%$ a $6,1 \%$. El Cuadro 4 da a conocer el movimiento que se tuvo con los países en términos de bajar de rango de disparidad, mantenerse en el mismo grupo de posiciones relativas, o bien, de avanzar en la clasificación.

Se tiene evidencia de que siete países mejoran en posiciones: Nicaragua, Uruguay, Honduras, Colombia, Panamá, Ecuador y Venezuela. También se muestra que seis países se mantuvieron en sus respectivos grupos de posición relativa: Brasil, Paraguay, Chile, Guatemala, El Salvador y Costa Rica. Este último país, se mantuvo en el rango alto, aún cuando la disparidad empeoró un $2 \%$.

Finalmente, cinco países empeoran su disparidad salarial en centros urbanos basada en diferencias de género, son ellos: México, Bolivia, Perú, República Dominicana y Argentina.

Mientras el Cuadro 4 detecta variaciones cualitativas pertenecientes a los rangos de los diferentes clusters o grupos, el Cuadro 5 especifica las diferencias que llegaron a ocurrir en

CUADRO 4

AMERICA LATINA Y EL CARIBE: MATRIZ COMPARATIVA

RESPECTO A DISPARIDADES EN SALARIOS URBANOS 1997-2006 BASADOS EN DIFERENCIAS DE GÉNERO

\begin{tabular}{|c|c|c|c|c|c|c|}
\hline & \multirow[b]{2}{*}{ AÑO } & \multicolumn{5}{|c|}{2006} \\
\hline & & BAJO & $\begin{array}{c}\text { MODERADO } \\
\text { BAJO }\end{array}$ & INTERMEDIO & $\begin{array}{c}\text { MODERADO } \\
\text { ALTO }\end{array}$ & ALTO \\
\hline \multirow{5}{*}{1997} & BAJO & $=\mathrm{Bra}$ & & & $\uparrow \mathrm{Nic}$ & \\
\hline & $\begin{array}{c}\text { MODERADO } \\
\text { BAJO }\end{array}$ & $\downarrow$ Mex & $\begin{array}{l}=\text { Par } \\
=\text { Chl } \\
=\text { Gua }\end{array}$ & $\uparrow$ Uru & & $\uparrow$ Hon \\
\hline & INTERMEDIO & $\downarrow \mathrm{Bol}$ & & & $\begin{array}{l}\uparrow \mathrm{Col} \\
\uparrow \mathrm{Pan}\end{array}$ & \\
\hline & $\begin{array}{l}\text { MODERADO } \\
\text { ALTO }\end{array}$ & $\downarrow$ Per & $\downarrow$ Red & & $=$ Els & $\begin{array}{l}\uparrow \mathrm{Ecu} \\
\uparrow \mathrm{Ven}\end{array}$ \\
\hline & ALTO & & & $\downarrow \operatorname{Arg}$ & & $=\mathrm{Cos}$ \\
\hline
\end{tabular}

Notas: $\downarrow$ bajan en su posición de 1997 a 2006; $\uparrow$ suben $y=$ mantienen igual categoría en el rango de agrupaciones; ARG: Argentina; BOL: Bolivia; BRA: Brasil; CHL: Chile; COL: Colombia; COS: Costa Rica; ECU: Ecuador; ELS: El Salvador; GUA: Guatemala; GUY: Guyana; HON Honduras; JAM: Jamaica; MEX: México; NIC: Nicaragua; PAN: Panamá; PAR: Paraguay; PER: Perú; RED: República Dominicana; TYT: Trinidad y Tobago; URU: Uruguay; VEN: Venezuela.

Fuente: Cálculos propios basados en la Comisión Económica para América Latina y el Caribe (CEPAL). América Latina frente al espejo: dimensiones objetivas y subjetivas de la inequidad social y el bienestar en la región. Santiago de Chile, Chile. CEPAL, 2010: 88. 


\section{CUADRO 5}

AMÉRICA LATINA:

VARIACIONES EN DISPARIDADES EN LOS SALARIOS URBANOS 1997-2006

BASADOS EN DIFERENCIAS DE GÉNERO

\begin{tabular}{|c|c|c|c|}
\hline GRUPO & PAÍS & $\begin{array}{c}\text { \% DE VARIACIÓN } \\
1997-2006\end{array}$ & $\begin{array}{l}\text { PROMEDIO APROXIMADO } \\
\text { DE GRUPO }\end{array}$ \\
\hline \multirow{5}{*}{ Alto } & Nicaragua & 20 & \multirow{5}{*}{14} \\
\hline & Honduras & 14 & \\
\hline & Brasil & 9 & \\
\hline & Chile & 9 & \\
\hline & Guatemala & 9 & \\
\hline \multirow{8}{*}{ Moderado alto } & Uruguay & 8 & \multirow{8}{*}{6} \\
\hline & Venezuela & 8 & \\
\hline & Colombia & 7 & \\
\hline & Ecuador & 7 & \\
\hline & Panamá & 6 & \\
\hline & El Salvador & 5 & \\
\hline & México & 4 & \\
\hline & Paraguay & 4 & \\
\hline Intermedio & Bolivia & 0 & 0 \\
\hline \multirow{2}{*}{ Moderado bajo } & Costa Rica & -2 & \multirow{2}{*}{-3} \\
\hline & Argentina & -5 & \\
\hline \multirow{2}{*}{ Baja } & República Dominicana & -6 & \multirow{2}{*}{-6} \\
\hline & Perú & -7 & \\
\hline
\end{tabular}

Fuente: Cálculos propios basados en Comisión Económica para América Latina y el Caribe (CEPAL). América Latina frente al espejo: dimensiones objetivas y subjetivas de la inequidad social y el bienestar en la región. Santiago de Chile, Chile. CEPAL, 2010: 88.

el ámbito de cada país. Como puede apreciarse, países que en general tienen indicadores bajos de desarrollo humano - tales son los casos de Nicaragua, Honduras y Guatemala- evidencian los más altos grados de mejora en las disparidades salariales. También en ese grupo se ubican Chile y Brasil.

Estos datos son alentadores si se toma en cuenta que entre los países más inequitativos del mundo, entre los primeros diez, los puestos del siete al diez son ocupados por (en ese orden): Haití, Bolivia, Colombia y Brasil. Ocho países mejoran también, no en el nivel alto, pero sí en el grupo moderado alto. Allí están los casos de Colombia, Paraguay, Venezuela, Uruguay y México, por ejemplo.

De conformidad con la ley de rendimientos decrecientes o de retornos disminuyentes, era de esperarse que quienes tienen mayor posibilidad de mejora, son las unidades de estudio, en este caso países, que tienen originalmente valores bajos. Es más difícil mostrar mejoras dramáticas o significativas a medida que se tienen ya valores altos.

Los casos de descenso que son más preocupantes, en los que la disparidad ha tendido 
a ser mayor, se refieren a Argentina, República Dominicana y Perú. En 2006, mientras en Venezuela las mujeres estarían a sólo un $6 \%$ de lograr la igualdad en el ingreso, esa distancia se manifiesta como la peor, en el caso de Perú, en donde la disparidad salarial se amplía a $23 \%$ de las mujeres, por debajo de los hombres.

También en esta fase de considerar las desigualdades salariales, se realizó un análisis por clusters o agrupaciones, cuyos resultados se tienen en la Figura 1. A medida que se avanza en la observación de la distancia 0 a 25 , se tiene respectivamente que las diferentes unidades de análisis - en este caso países - tienen una separación individual, o bien, en la distancia 25 , formando un solo grupo. Las agrupaciones más útiles pueden observarse alrededor de la distancia 5 .

FIGURA 1

INEQUIDAD EN 2006

RESULTADOS DE CLUSTER ANÁLISIS

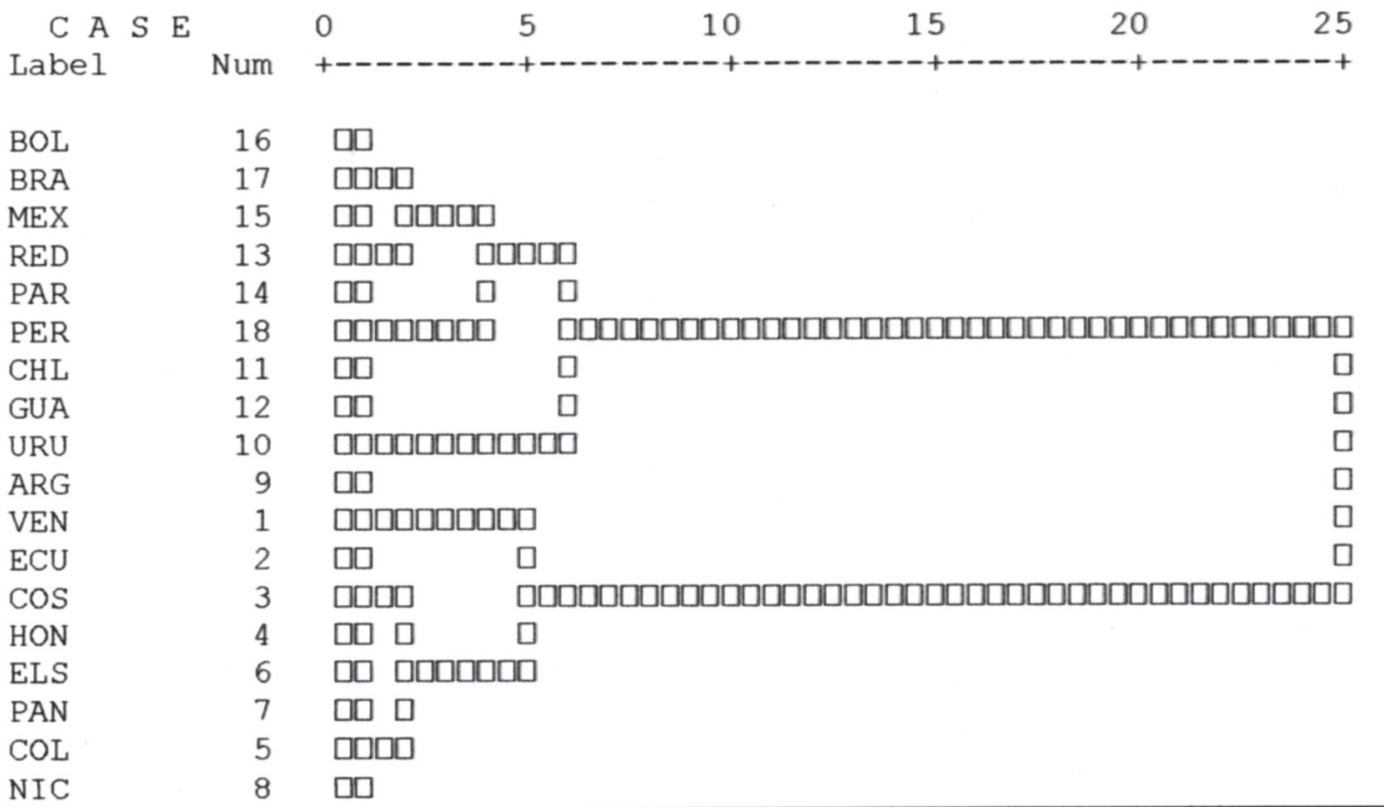

Fuente: Cálculos propios, con base en datos de CEPAL.

Como se aprecia, Brasil, México y República Dominicana formarían un grupo, que luego se enlaza con otro, formado por Chile, Guatemala y Uruguay. Argentina y Nicaragua se identifican más bien con particularidades muy propias, situación que es similar en el caso de Perú. Nicaragua presenta valores altos, en tanto que Perú y Argentina evidencian condiciones, en las cuales se tuvo mayor desigualdad en los salarios de mujeres respecto a hombres.
Hasta aquí, una primera etapa de los resultados en los cuales se concentró la atención en el estudio de las diferencias salariales.

A continuación, se realizó la consideración del crecimiento económico. Para ello se estableció el crecimiento económico acumulado de los diferentes países de la región de 2000 a 2009. El Cuadro 6 muestra esos resultados.

De ese cuadro es posible establecer que el grupo alto está conformado por Trinidad y 


\section{CUADRO 6 \\ AMÉRICA LATINA 2000-2009: \\ CRECIMIENTO ECONÓMICO ACUMULADO}

Y ELASTICIDAD DE DESIGUALDAD SALARIAL DE MUJERES SOBRE CRECIMIENTO

\begin{tabular}{|c|c|c|c|}
\hline GRUPO & PAÍS & $\begin{array}{l}\text { CRECIMIENTO } \\
\text { ACUMULADO } \\
(\%) \\
\end{array}$ & $\begin{array}{c}\text { CLASIFICACIÓN DE } \\
\text { CRECIMIENTO POR RANGO } \\
\text { INTEGRADO }{ }^{1}\end{array}$ \\
\hline \multirow{2}{*}{ Alto } & Trinidad y Tobago & 67,6 & 0,997 \\
\hline & Panamá & 58,2 & 0,842 \\
\hline \multirow{3}{*}{ Moderado alto } & Perú & 51,2 & 0,726 \\
\hline & República Dominicana & 50,6 & 0,716 \\
\hline & Belice & 48,5 & 0,682 \\
\hline \multirow{11}{*}{ Intermedio } & Honduras & 42,9 & 0,589 \\
\hline & Ecuador & 42,4 & 0,581 \\
\hline & Suriname & 40,6 & 0,551 \\
\hline & Costa Rica & 40,4 & 0,548 \\
\hline & Colombia & 39,7 & 0,536 \\
\hline & Venezuela & 39,6 & 0,535 \\
\hline & Bolivia & 37,0 & 0,492 \\
\hline & Chile & 36,3 & 0,480 \\
\hline & Argentina & 35,6 & 0,469 \\
\hline & Guatemala & 33,5 & 0,434 \\
\hline & Brazil & 33,4 & 0,432 \\
\hline \multirow{5}{*}{ Moderado bajo } & América Latina y Caribe & 31,2 & 0,396 \\
\hline & Uruguay & 29,5 & 0,368 \\
\hline & Nicaragua & 28,8 & 0,356 \\
\hline & Paraguay & 23,0 & 0,261 \\
\hline & El Salvador & 22,4 & 0,251 \\
\hline \multirow{4}{*}{ Bajo } & México & 19,1 & 0,196 \\
\hline & Guyana & 15,4 & 0,135 \\
\hline & Jamaica & 9,4 & 0,036 \\
\hline & Haití & 7,3 & 0,002 \\
\hline
\end{tabular}

Nota 1: Rango integrado: estableciendo distribución en todo el rango original con ubicación de percentiles.

Fuente: Cálculos propios con base en datos de CEPAL y de Cuadros 3 y 5 de este estudio.

Tobago junto con Panamá; este grupo tiene un promedio de $63 \%$ de crecimiento acumulado. En el grupo moderado alto están tres países: Perú, República Dominicana y Belice, con un promedio acumulado de 50\%. En el grupo intermedio tenemos a la mayor parte de países, con un promedio de 38\%. En el rango de moderado bajo tenemos un promedio de
27\% y este grupo está integrado por Uruguay, Nicaragua, El Salvador y Paraguay, además del promedio regional. En el grupo bajo se encuentran México, Guyana, Jamaica y Haití, en ellos el promedio es de $13 \%$.

En una tercera parte del tratamiento de datos -en primera fue la consideración exclusiva de las desigualdades salariales, en tanto en 
la segunda se abordó la variable de crecimiento económico- se establece una comparación entre desigualdades y crecimiento.

Aquí, en el Cuadro 7 se dan a conocer los resultados de las elasticidades, es decir, que porcentaje de desigualdad disminuyó (signo positivo) o aumentó (signo negativo) de la proporción de pagos a mujeres respecto a hombres, en función de un $1 \%$ de crecimiento económico - dado que aunque en algunos casos los niveles fueron bajos, todos los países mostraron un crecimiento de producción positivo en la primera década del siglo XXI.

\section{CUADRO 7}

AMÉRICA LATINA 2000-2009: ELASTICIDAD DE DESIGUALDAD SALARIAL DE MUJERES SOBRE CRECIMIENTO ECONÓMICO

\begin{tabular}{ccc}
\hline GRUPO & PAÍS & ELASTICIDAD $^{1}$ \\
\hline Alto & Nicaragua & 0,694 \\
\hline Intermedio & Honduras & 0,326 \\
\hline & Uruguay & 0,271 \\
& Brazil & 0,269 \\
Moderado bajo & Guatemala & 0,269 \\
& Chile & 0,248 \\
& El Salvador & 0,223 \\
& México & 0,209 \\
& Venezuela & 0,202 \\
& Colombia & 0,176 \\
& Paraguay & 0,174 \\
& Ecuador & 0,165 \\
\hline & Panamá & 0,103 \\
& Bolivia & 0,000 \\
& Costa Rica & $-0,050$ \\
& Argentina & $-0,119$ \\
& Perú & $-0,137$ \\
& & $-0,140$ \\
\hline
\end{tabular}

Nota 1: Elasticidad de desigualdad salarial de mujeres respecto a hombres sobre crecimiento económico. Fuente: Cálculos propios con base en datos de CEPAL y de Cuadros 3 y 5 de este estudio.

En el caso de que Nicaragua y Honduras encabecen la lista de mayores elasticidades positivas, tomando en cuenta equidad y crecimiento económico, conviene tener presente que - aunque ambas naciones no tienen un dramático crecimiento económico- lo que más afecta es la menor desigualdad, que según cifras oficiales, se ha efectuado en los salarios entre mujeres $y$ hombres.
Se puede notar que el valor alcanzado por Nicaragua es tan grande que en la distribución por quintiles eliminó el grupo moderado alto. Esto se debe a que esta forma de agrupación, al igual que la media aritmética, se encuentra muy afectada por los valores extremos. Una situación diferente se presenta al considerar la moda o la mediana como valores de centralidad. 
De manera complementaria, se realizó un análisis utilizando coeficientes de correlación (véase Anexo 1) y modelo de regresión múltiple (véase Anexo 2). En el primero de los casos de las variables estudiadas - datos de desigualdad salarial de 1997, de desigualdad salarial 2006 y de crecimiento económico acumulado- no se encontraron cifras de correlación significativas.

En cuanto a los datos de regresión lineal, el valor de la variable exógena -inequidad 2006 , siendo la variable dependiente crecimiento económico - no se determinaron influencias significativas ni a 1 ni a $5 \%$ de error; la significancia muestra un $6 \%$ en el margen de error, lo que se manifestaría como significativa si se amplía el nivel de confianza a 10\% (véase valores y gráfico en Anexo 2).

Los anexos 3 y 4 dan a conocer que sí se tenía normalidad en la distribución de residuos. Eso se da a conocer en la gráfica del anexo 3, que establece comparación con la curva normal $y$ en el anexo 4, en donde se observa el comportamiento de los residuos con los límites de distribución que se sigue considerando un patrón de normalidad.

\section{CONSIDERACIONES FINALES Y CONCLUSIONES}

Las disparidades entre salarios de hombres $y$ mujeres, en donde ambos presentan condiciones comparables de preparación o capacitación $y$ desempeño productivo, han mejorado en Latinoamérica, al pasar de $80 \%$ en 1997 a 85\% como promedio regional en el 2006. Para este último año, las naciones que mejores estándares tenían -las mujeres en sus ingresos respecto a los hombres, con una situación que no llega aún a la igualdad- son: Venezuela, Ecuador, Honduras y Costa Rica. No se evidencian resultados en función de que las economías grandes -Argentina, Brasil y México- tengan menos disparidad que las economías de pequeños mercados domésticos relativos.

Los países en los cuales las condiciones de ingreso para las mujeres son las de menor proporción respecto a los hombres, para el 2006, son: México, Bolivia, Brasil y Perú. En este grupo, el promedio de ingreso para las mujeres es de 79\%. El grupo de menor disparidad tiene $93 \%$.

Los países que desde 1997 tienden a mejorar su situación de disparidad respecto a los ingresos son: Nicaragua, Honduras y Guatemala, además de Chile y Brasil. Estas naciones se ven favorecidas por los niveles que presentaban en un inicio, con valores relativamente bajos, lo que hace que con mayor facilidad puedan disminuir sus disparidades, dada la ley de retornos disminuyentes o de incrementos decrecientes.

Las mayores tasas de crecimiento económico durante la primera década del siglo XXI se llegaron a tener en Trinidad y Tobago y Panamá; de una manera ampliada se incluiría a Perú, República Dominicana y Belice. Las tasas de menor crecimiento acumulado se evidenciaron en Guyana, Jamaica y Haití. Este último país no solo ha tenido un índice de desarrollo bajo y ha sido el país menos desarrollado económicamente en el hemisferio occidental, sino que el terremoto del 12 de enero de 2010 ha devastado la aminorada infraestructura del país.

Más que por la influencia de crecimiento económico, la repercusión directa de la mejora en cuanto a disminuir las desigualdades salariales entre hombres y mujeres, es lo que hace que Nicaragua y Honduras sean las naciones, en las cuales la elasticidad de desigualdad de salarios sobre crecimiento de producción, adquieran los valores más altos. Los países en los cuales esas elasticidades mostraron signos negativos son especialmente Perú y Argentina.

\section{BIBLIOGRAFÍA}

Arcand, Jean y D'Hombres, Beatrice. Racial discrimination in the brazilian labour market: wage, employment and segregation effects. Clermont-Ferrand, Francia: Université d'Auvergne, 2004.

Barrera, Dalia y Aguirre, Irma. "Liderazgos femeninos y políticas públicas a favor de las mujeres en gobiernos locales en México". Dalia Barrera Bassols y Alejandra Massolo (comps.). El municipio. Un reto para la igualdad de oportunidades entre hombres y mujeres. 
México, DF: GIMTRAP/Inmujeres/PNUD, 2003.

Comisión Económica para América Latina y el Caribe (CEPAL). Panorama social de América Latina 2009. Santiago de Chile, Chile: CEPAL, 2009.

Comisión Económica para América Latina y el Caribe (CEPAL). América Latina frente al espejo: dimensiones objetivas y subjetivas de la inequidad social y el bienestar en la región. Santiago de Chile, Chile: CEPAL, 2010.

Darnou, Amanda. Gender in policy and practice. New York: Routledge Farlner, 2002.

Demurger, Sylvie; Fournier, Martin y Yi, Chen. The evolution of gender earnings gaps and discrimination in urban China: 1988-1995. Hong Kong: Society for the Study of Economic Inequality, University of Hong Kong, 2005.

Dong, Xiaoyuan et ál. "Gender segmentation at work in China's privatizad rural industry: some evidence from Shandong and Jiangsu". World Development 32 (6). 2004: 979-998.

Esteve-Volart, Berta. Gender discrimination and growth: theory and evidence from India. London: London School of Economics and Political Science, 2004.

Gill, Judith. Beyond the great divide. Sydney, Australia: University of New South Wales Press, 2004.

Graham, Carol. "Mobility, opportunity and vulnerability: the dynamics of poverty and inequality in a global economy". Journal of Human Development 3 (1). New York: UNDP, 2002.
Ho, Samuel et ál. "Privatization and enterprise wage structures during transition: evidence from rural industry in China". Economics of Transition 10 (3). November 2002: 659-688.

Klein, Susan. Achieving gender equity. Mahwah, New Jersey: Lawrence, Eribaum Inc., 2007.

Krieger, Nancy. Emboying inequality. London: International Health Services, 2005.

Massolo, Alejandra. "Introducción. Política y mujeres: una peculiar relación”. Alejandra Massolo (comp.). Los medios y los modos: participación política y acción colectiva de las mujeres. México, DF: El Colegio de México, 1994.

Massolo, Alejandra. Gobiernos locales y mujeres: nuevos cambios y desafíos en América Latina. México, DF: UNRISD, 2005.

Meng, Xin. "Occupational segregation and its impact on gender wages discrimination in China's rural industrial sector". Oxford Economic Papers 47 (1). January 1995:136-155.

Romesburg, Charles. Cluster analysis for researchers. London: Routledge, 2004.

Rozelle, Scott. "Gender wage gaps in postreform rural China". Pacific Economic Review 7 (1). 2002: 157-179.

Salomone, Rosemary. Same, different, equal. New Haven, New Jersey: Yale University Press, 2005.

UNDP. Women's political participation and good gobernance: 21st. Century challenges. New York: UNDP/UNO, 2001.

Fecha de ingreso: 30/08/2010

Fecha de aprobación: 14/03/2011 


\section{ANEXOS}

ANEXO 1

MATRIZ GENERAL DE COEFICIENTES DE CORRELACIÓN ENTRE VARIABLES: INEQUIDAD EN 1997 Y 2006 / CRECIMIENTO ECONÓMICO ACUMULADO 2000-2009

\begin{tabular}{|c|c|c|c|c|}
\hline & & INEQ06 & INEQ97 & CREAC006 \\
\hline \multirow{3}{*}{ INEQ06 } & $\begin{array}{l}\text { Correlación de } \\
\text { Pearson }\end{array}$ & 1,000 & ,372 & ,179 \\
\hline & Sig. (bilateral) & , & ,128 & ,477 \\
\hline & $\mathrm{N}$ & 18 & 18 & 18 \\
\hline \multirow{3}{*}{ INEQ97 } & $\begin{array}{l}\text { Correlación de } \\
\text { Pearson }\end{array}$ & ,372 & 1,000 & ,446 \\
\hline & Sig. (bilateral) & ,128 & , & ,064 \\
\hline & $\mathrm{N}$ & 18 & 18 & 18 \\
\hline \multirow{3}{*}{ CREAC006 } & $\begin{array}{l}\text { Correlación de } \\
\text { Pearson }\end{array}$ & ,179 & ,446 & 1,000 \\
\hline & Sig. (bilateral) & ,477 &, 064 & , \\
\hline & $\mathrm{N}$ & 18 & 18 & 18 \\
\hline
\end{tabular}

Fuente: Cálculos propios, con base en datos de CEPAL. 
ANEXO 2

MODELO DE REGRESIÓN LINEAL

INEQUIDAD EN 2006 Y CRECIMIENTO ECONÓMICO ACUMULADO 2000-2009

\begin{tabular}{|c|c|c|c|c|c|}
\hline \multicolumn{6}{|c|}{$\begin{array}{l}\text { ANOVA Table } \\
\text { crecac } 0009 \text { vs. ineq } 2006\end{array}$} \\
\hline & DF & Sum of Square: & Mean Square & F-Vahue & P-Vahue \\
\hline \multirow{3}{*}{$\begin{array}{l}\text { Regression } \\
\text { Residual } \\
\text { Total }\end{array}$} & 1 & 356,490 & 356,490 & 3,936 &, 0647 \\
\hline & 16 & 1449,288 & 90,581 & & \\
\hline & 17 & 1805,778 & & & \\
\hline
\end{tabular}

Regression Coefficients crecac 0009 vs. ineq 2006

\begin{tabular}{l|r|r|r|r|r|} 
& \multicolumn{1}{c}{ Coefficient } & \multicolumn{1}{c}{ Std. Error } & \multicolumn{1}{c}{ Std Coeff. } & \multicolumn{1}{c}{ t-Vahe } & P-Vahre \\
\cline { 2 - 6 } Intercept & $-16,301$ & 26,906 & $-16,301$ &,- 606 &, 5531 \\
\cline { 2 - 7 } ineq2009 &, 662 &, 334 &, 444 & 1,984 &, 0647 \\
\cline { 2 - 6 } & & &
\end{tabular}

\section{Regression Plot}

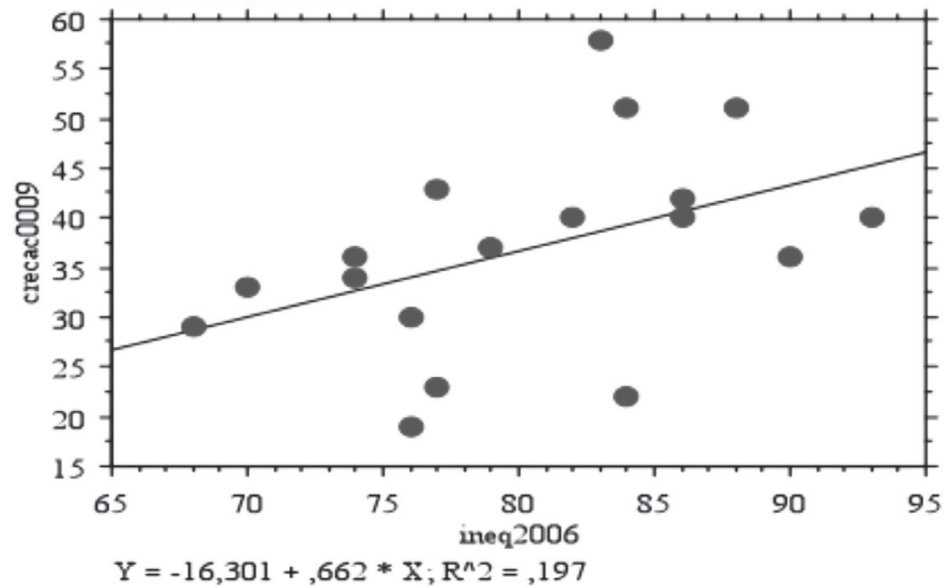

Fuente: Cálculos propios, con base en datos de CEPAL. 
ANEXO 3

MODELO DE REGRESIÓN LINEAL

PRUEBA NO PARAMÉTRICA $\left(\mathrm{X}^{2}\right)$ PARA COMPROBAR NORMALIDAD

EN LA DISTRIBUCIÓN DE RESIDUOS

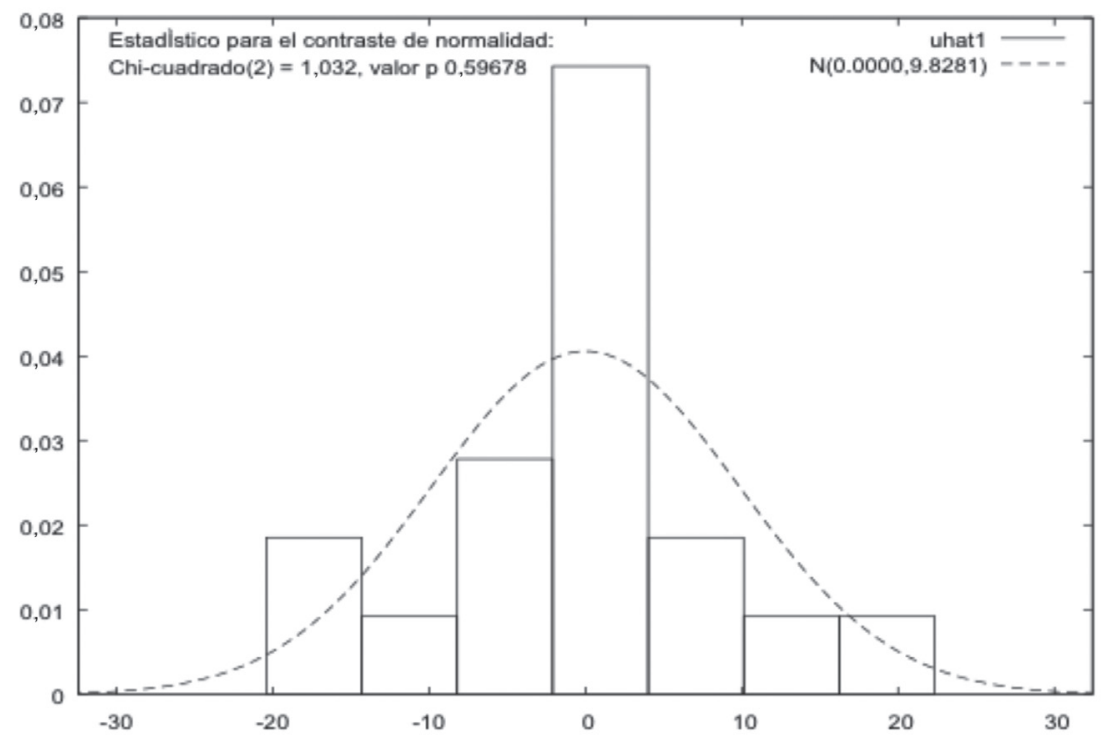

Fuente: Cálculos propios, con base en datos de CEPAL. 


\section{ANEXO 4 \\ MODELO DE REGRESIÓN LINEAL \\ GRÁFICA CUSUM DE RESIDUOS DENTRO DE LÍMITES DE NORMALIDAD}

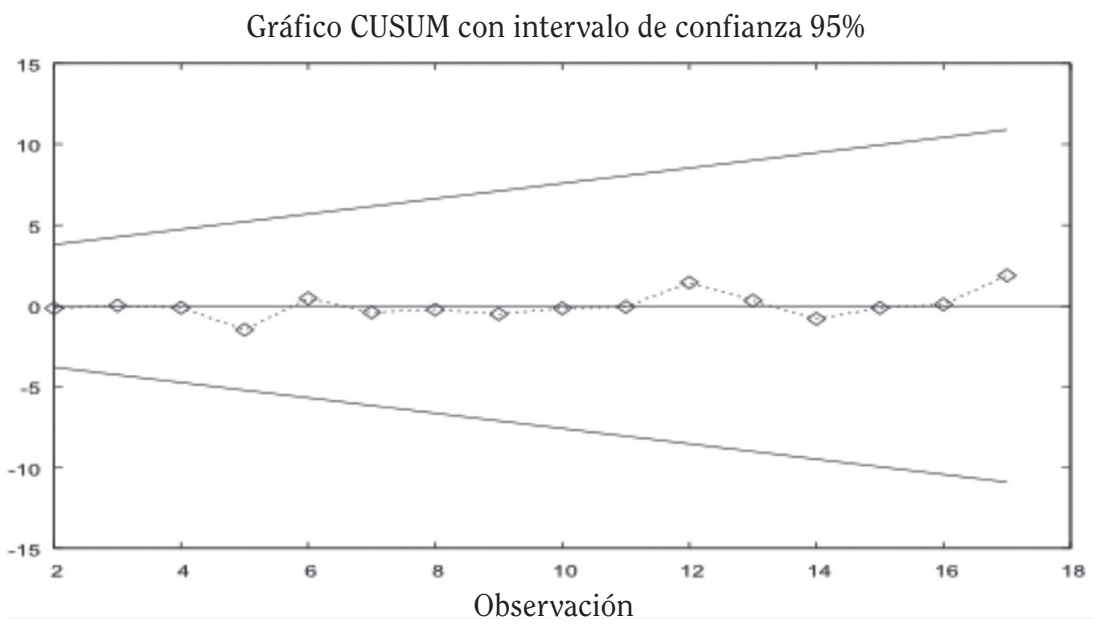

Fuente: Cálculos propios, con base en datos de CEPAL. 